\title{
Pre-stack Time Migration Processing for Block A of Putaohua Oilfield
}

\author{
Wang zhiyuan ${ }^{1, a}$ \\ ${ }^{1}$ Northeast petroleum university, Daqing, Heilongiiang, China
}

\begin{abstract}
According to the reservoir and structural characteristics of Heidimiao, this paper combines fine pre-stack pre-processing and small-panel pre-stack time migration, and optimizes targeted methods and parameters to obtain the seismic data with higher resolution and better amplitude retention. Compared with the previous processing results, the migration imaging of the new data has been improved, and the geological phenomenon of the whole area is clear. The fracture system is reasonable, the fracture points are crisp, and the imaging of small faults is clear. The wave group characteristics of the new treatment are obvious, and the transverse resolution, longitudinal resolution and signal-to-noise ratio are improved obviously. By using relative amplitude preserving and VTI pre-stack time migration, the signal to noise ratio and resolution of seismic processing results have been greatly improved, and the interlayer information is more abundant, which can meet the needs of target interpretation.
\end{abstract}

\section{Introduction}

In seismic exploration, the phenomenon that the position of the reflection point of the stratum with a certain dip shown on the horizontal superimposed section deviates from the true position of the underground reflection point is called offset. The offset location of seismic section is to locate the offset reflection layer on the horizontal superposition section, so as to restore the true shape of the underground reflection layer, which is often called "migration"[1]. Migration can improve the resolution of imaging, accurately display the wavelet shape and various parameters, make the formation structure, lithology and stratigraphic traps and other contents more accurate and fine, improve the accuracy of reservoir prediction, and provide a reliable and comprehensive reference data for oil exploitation ${ }^{[2]}$.

In specific exploration practice, when the formation is flat, horizontal stacking can meet the requirements; when the formation is steep, the overlap of the common center point causes the reflection point to be blurred, and the pre-stack migration can achieve true common reflection point imaging. The prestack time migration is the best choice when the lateral velocity variation is small and the underground structure is complex. However, prestack depth migration is more suitable for complex structure and large lateral variation of velocity field. However, most of the commonly used pre-stack migration methods are used in the case of homogeneous and isotropic underground media, but cannot be used in the case of complex anisotropy in actual underground media, and even the imaging error of underground structure may occur. Therefore, in order to solve this problem, the imaging technology of anisotropic medium wave field is introduced.

\section{Kirchhoff integral migration based on VTI medium}

At present, although most seismic data processing methods assume that the underground medium is isotropic, many studies have proved that most rocks are anisotropic. With the development of seismic exploration technology, the influence of the anisotropy of underground media on seismic wave velocity has become an important factor in determining the accuracy of seismic exploration ${ }^{[3]}$.

In the past, when dealing with seismic data, the influence of velocity anisotropy was often attributed to velocity errors. Nowadays, the resolution and imaging quality of seismic data are increasingly required. In particular, reservoir prediction and reservoir description require high resolution and fidelity of seismic data, as well as clear fault planes to determine the role of faults in hydrocarbon migration and sealing. Therefore, velocity anisotropy has become one of the main obstacles to further improve the resolution of seismic exploration and imaging therapy. The application of anisotropic pre-stack time migration technology based on VIT media not only can eliminate the influence of dielectric anisotropy on the imaging accuracy, makes the breakpoints, cross section and the contact relationship between layer more clearly, and improve the reservoir, shallow in the small fault prediction accuracy of distribution, but also can solve the problem of excessive correction of pre-stack inversion

2011yzdwzy@sina.com 
common reflection point (CRP) gather cannon offset data. This technology has been applied in the middle and shallow oil and gas exploration in the Songliao Basin in the Daqing exploration area, and has produced very good results.

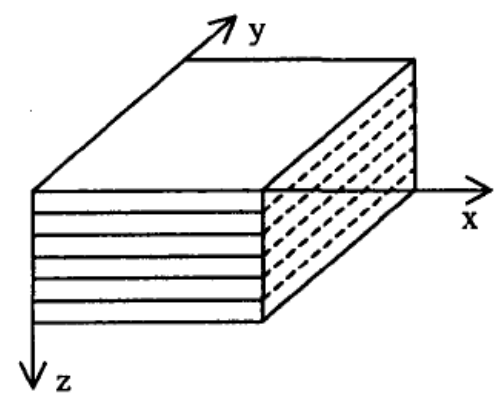

Fig. 1 Schematic diagram of VTI medium-periodic thin intersedimentary rock

Kirchhoff integration method migration is a commonly used method in actual seismic data processing. The theoretical basis of the Kirchhoff integration method is the Kirchhoff decomposition of the wave equation, which uses the Kirchhoff integration formula to reversely extrapolate the seismic data recorded on the surface to achieve migration imaging. VIT (transversely isotropic media with a vertical symmetry axis, abbreviated as VIT) medium is a transversely isotropic medium with a vertical symmetry axis. Kirchhoff integral method and finite difference method are generally used in prestack time migration, and the integral method is the main method. The integral method uses Kirchhoff product decomposition of wave equation to carry out wave field extrapolation, and on the basis of Kirchhoff integral migration combined with ray theory to carry out migration velocity analysis. In Kirchhoff integral prestack migration imaging, the imaging point underground is usually regarded as a possible diffraction point, and the travel time and amplitude factor from the source point to the imaging point and then to the receiving point are calculated by the ray theory under the condition of high frequency approximation, and then the seismic trace within the set aperture is superpositioned according to the calculated travel time and amplitude factor.

The anisotropic prestack time migration is based on the anisotropy theory to calculate travel time ${ }^{[4]}$. For P-wave anisotropic media, the travel time formula in VTI media derived by Alkhalifah is:

$$
t\left(\tau, x, x_{0}, h, v, \eta\right)=\frac{1}{2} p_{g} y_{g}+\frac{1}{2} p_{s} y_{s}+\frac{\tau}{2}\left(\sqrt{1-\frac{v^{2} p_{g}^{2}}{1-2 v^{2} \eta p_{g}^{2}}}+\sqrt{1-\frac{v^{2} p_{s}^{2}}{1-2 v^{2} \eta p_{s}^{2}}}\right)
$$

Where: $t$ is the travel time; $v$ is the root mean square velocity of the overlying layer of the reflection point; $\tau$ is the two-way vertical travel time of the reflection point; $\mathrm{h}$ is the half offset; $x_{s}$ is the coordinates of the reflection point; $x$ is the inspection center point coordinates; $\eta$ is anisotropic parameters; $y_{s}$ and $y_{s}$ are the lateral distances from the reflection point to the shot point and the receiver point, respectively; $p_{s}$ and $p_{g}$ are the ray parameters of the seismic source and receiver point. According to Alkhalifah and Tsvankin's velocity analysis and research on the transverse homogeneous medium, the subscript $\mathrm{s}$ and $\mathrm{g}$ are slightly divided, and the ray parameter $\mathrm{p}$ can be expressed as:

$$
p^{2}=p_{i s o}^{2} \times \frac{y^{6}+6 v^{2} y^{4}(1-\eta) \tau^{2}+3 v^{4} y^{2}(3+4 \eta)+4 v^{6} \tau^{6}}{y^{6}(1+2 \eta)+2 v^{2} y^{4}(3+5 \eta) \tau^{2}+v^{4} y^{2}(9+4 \eta) \tau^{4}+4 v^{6} \tau^{6}}
$$

Where

$$
p_{\text {iso }}^{2}=\sqrt{\frac{y^{2}}{v^{2} y^{2}+v^{4} \tau^{4}}}
$$

It can be seen from the above formula that the travel time of compressional wave in anisotropic medium can be calculated as long as two parameters $v$ and $\eta$ are known. When $\eta=0$, $\mathrm{p}$ is simplified to $p_{i s o}$ in the isotropic medium.

\section{Practical data processing}

The realization process of anisotropic pre-stack time migration is more complicated than that of isotropic prestack time migration. It needs to obtain the $\eta$ field on the basis of accurately obtaining the root mean square velocity. The specific realization process is as follows:

(1) Pre-processing: The gathers subjected to pre-stack time migration processing should be pre-processed with static correction, pre-stack denoising, energy compensation, deconvolution, and residual static correction. A higher signal-to-noise ratio and spatially more balanced amplitude energy are the basis for a good pre-stack time migration.

(2) Establish two initial fields: namely, establish the initial root mean square velocity field and the medium anisotropy parameter $\eta$ field.

(3) Target line migration: perform anisotropic prestack time migration processing for the target line, output CRP gathers, and perform quality control.

(4) Velocity field and $\eta$ field optimization: Optimize the root mean square velocity field and the medium anisotropy parameter $\eta$ field, so that the in-phase axis of the output CRP gather is leveled.

(5) Iterative offset of target line: repeat steps (3) and (4) to iteratively process the target line, until the output CRP gather all offsets and phase axis are leveled to terminate the iteration.

(6) Full data volume migration: Perform anisotropic pre-stack time migration for the entire $3 \mathrm{D}$ seismic data volume.

\section{Treatment effect analysis}

According to the requirements of geological tasks and processing, through analyzing the original data, this paper determines the processing difficulties in this area, adopts targeted processing measures, carries out a large number of trial processing tests, determines the processing process and carries out batch processing. The profile of this processing has the following characteristics:

(1) From the overall effect of the pre-stack time migration result profile processed this time, it can be seen that the signal-to-noise ratio is moderate, the resolution is high, the waveform is natural, the horizon is complete, and 
the wave group characteristics of each reflection target layer are more prominent. The information is richer, and the overall effect is coordinated and unified. The diffracted waves converge, the breakpoints and cross-sections are clear, the structure is accurately imaged, and the base shape is prominent.

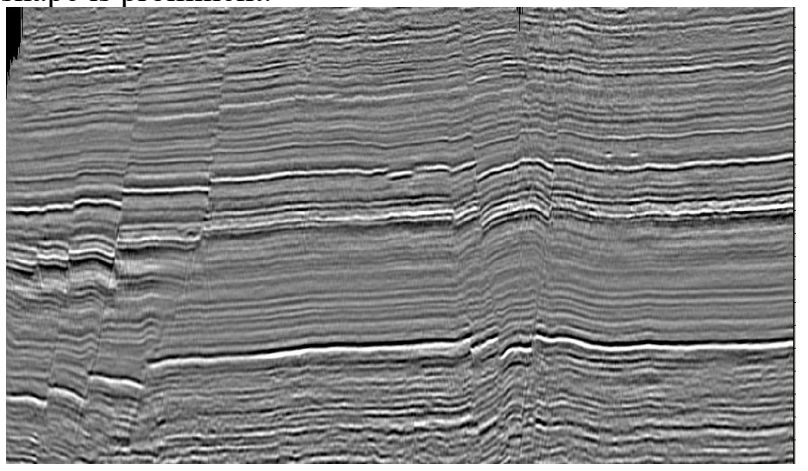

Fig. 2 Pre-stack time migration profile (Crossline 1880 line)

(2) Comparing the time slices of the pre-stack time migration and post-stack time migration profiles, it can be seen that the signal-to-noise ratio of the pre-stack time migration profile is relatively high, the wave group characteristics are relatively clear, the inter-layer information is rich, and the diffraction wave Convergence in place, the imaging of cross-sections and steep dip angles is clearer.

(3) Through spectrum analysis and filtering scanning of the final results, it can be concluded that the effective signal frequency band range of the final data is: T06 reflection segment: $8-140 \mathrm{~Hz}$; T1 reflection section: 8 $110 \mathrm{~Hz} ; \mathrm{T} 2$ reflection section: $8-90 \mathrm{~Hz}$.

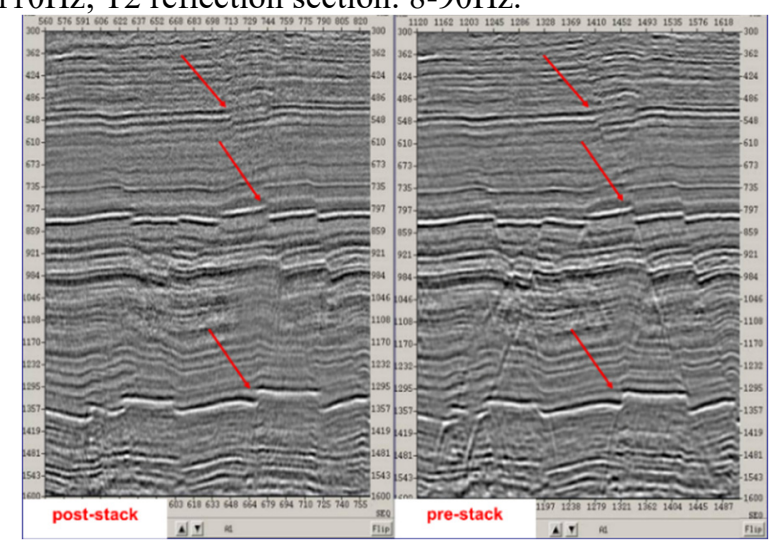

Fig. 3 Profile comparison of post-stack and pre-stack time migration (Inline2158 line)

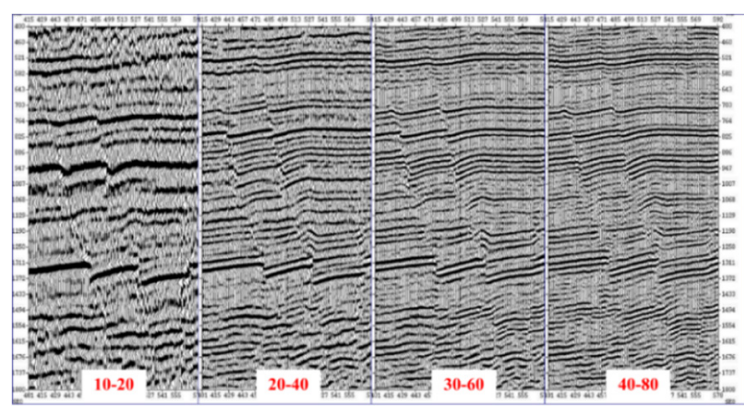

Fig. 4 Band-pass filtering scan of the product profile

\section{Conclusion}

The design of the seismic data processing flow in this paper is reasonable, the parameters are selected appropriately. And the final result profile has a moderate signal-to-noise ratio, high resolution, natural waveforms, obvious wave group characteristics, sufficient interlayer information, clear breakpoints and the structure of sections, special geological bodies and basements is clear. In the final result, the apparent dominant frequency of the T06 reflective layer reaches $70 \mathrm{~Hz}$, the apparent dominant frequency of the T1 reflective layer reaches $60 \mathrm{~Hz}$, and the apparent dominant frequency of the $\mathrm{T} 2$ reflective layer reaches $55 \mathrm{~Hz}$. Therefore, it can meet the requirements of geological missions.

Comprehensive analysis, the author believes that this treatment has the following characteristics:

(1) The combination of tomography and micro-logging statics solves the field statics problem, and the multiple iterations of velocity analysis and residual statics eliminate the influence of residual statics, which lays a foundation for obtaining accurate stacking velocity and establishing velocity field of prestack time migration.

(2) The signal-noise separation technology is used to effectively separate the front wave interference, and the subtraction method is used for attenuation; for $50 \mathrm{~Hz}$ industrial electricity, the single-frequency adaptive noise attenuation method is used to suppress it. While suppressing the noise effectively, the effective wave is protected to the greatest extent.

(3) Spherical diffusion compensation and surface consistent amplitude compensation can effectively solve the problems of longitudinal compensation and transverse equalization of energy.

(4) The methods of surface consistent deconvolution and predictive deconvolution combination are applied before stack to improve the longitudinal resolution of the target layer and highlight the wave group characteristics of each reflection layer, so that the result profile is consistent as a whole.

(5) The prestack time migration method based on VTI medium is adopted to solve the problems of complex structure and fault imaging, improve the imaging accuracy of the target layer, and make the fault and fault imaging accurately.

\section{References}

1. Lu, J.(1982)Principles of Seismic Exploration. Petroleum Industry Press,Beijing.

2. Zhang, Y.(2020) Analysis on the application of 3-D seismic pre-stack time migration processing technology. Petrochemical Technology. 2020,27(08):70-71.

3. Li, M. Li, Z. Ma, C. Duan, C. Li, H. (2010) Fine sand body contrast of Lower Crataceous Heidimiao reservoir under the constrained seismic inversion in southern Aonan Oilfield. Lithology reservoir. 2010,22(S1):74-79.

4. Su, L. Wang, J. Feng, W. Na, X. (2010)Application of 
Anisotropic Prestack Time Migration Technology in Daqing Exploration Area. Oil Geophysical Prospecting. 2010,45(S1):71-73+239+249. 